International Journal of Modern Physics A

(C) World Scientific Publishing Company

\title{
Early Universe Sources for CMB Non-Gaussianity
}

\author{
ALEJANDRO GANGUI \\ Instituto de Astronomía y Física del Espacio, 1428 Buenos Aires, Argentina, and \\ Departamento de Física, Universidad de Buenos Aires, 1428 Buenos Aires, Argentina. \\ Received (Day Month Year) \\ Revised (Day Month Year)
}

\begin{abstract}
In the framework of inflationary models with non-vacuum initial states for cosmological perturbations, we study non-Gaussian signatures on the cosmic microwave background (CMB) radiation produced by a broken-scale-invariant model which incorporates a feature at a privileged scale in the primordial power spectrum.
\end{abstract}

Keywords: cosmic microwave background; inflation.

\section{Introduction}

The common belief that the CMB is Gaussian distributed can be directly traced back to the generic assumption that the quantum fluctuations of the inflaton field are placed in the vacuum state 1. Relaxing this assumption might lead to detectable signatures in various astrophysical tests, most interestingly in future CMB and large-scale structure observations. In this note, we study CMB non-Gaussian signatures predicted within inflationary models with non-vacuum initial states for cosmological perturbations. The model incorporates a privileged scale, which implies the existence of a feature in the primordial power spectrum. The model predicts a vanishing three-point correlation function for the CMB temperature anisotropies 2 . We here focus on the first non-vanishing moment, the CMB four-point function at zero lag, namely the kurtosis, and compute its expected value for different locations of the primordial feature in the spectrum, as suggested in the literature to conform to observations of large scale structure 34 .

\section{Two-point correlation function for non-vacuum initial states}

We consider non-vacuum states for the cosmological perturbations of quantum mechanical origin. Let $\mathcal{D}(\sigma)$ be a domain in momentum space, such that if $\mathbf{k}$ is between 0 and $\sigma$, the domain $\mathcal{D}(\sigma)$ is filled by $n$ quanta, while otherwise $\mathcal{D}$ contains nothing. The state $\left|\Psi_{1}(\sigma, n)\right\rangle$ is defined by

$$
\left|\Psi_{1}(\sigma, n)\right\rangle \equiv \prod_{\mathbf{k} \in \mathcal{D}(\sigma)} \frac{\left(c_{\mathbf{k}}^{\dagger}\right)^{n}}{\sqrt{n !}}\left|0_{\mathbf{k}}\right\rangle \bigotimes_{\mathbf{p} \notin \mathcal{D}(\sigma)}\left|0_{\mathbf{p}}\right\rangle=\bigotimes_{\mathbf{k} \in \mathcal{D}(\sigma)}\left|n_{\mathbf{k}}\right\rangle \bigotimes_{\mathbf{p} \notin \mathcal{D}(\sigma)}\left|0_{\mathbf{p}}\right\rangle .
$$


The state $\left|n_{\mathbf{k}}\right\rangle$ is an $n$-particle state satisfying, at conformal time $\eta=\eta_{\mathrm{i}}: c_{\mathbf{k}}\left|n_{\mathbf{k}}\right\rangle=$ $\sqrt{n}\left|(n-1)_{\mathbf{k}}\right\rangle$ and $c_{\mathbf{k}}^{\dagger}\left|n_{\mathbf{k}}\right\rangle=\sqrt{n+1}\left|(n+1)_{\mathbf{k}}\right\rangle$. We have the following property

$$
\left\langle\Psi_{1}(\sigma, n) \mid \Psi_{1}\left(\sigma^{\prime}, n^{\prime}\right)\right\rangle=\delta\left(\sigma-\sigma^{\prime}\right) \delta_{n n^{\prime}} .
$$

It is clear from the definition of the state $\left|\Psi_{1}\right\rangle$ that the transition between the empty and the filled modes is sharp. In order to "smooth out" the state $\left|\Psi_{1}\right\rangle$, we consider a state $\left|\Psi_{2}\right\rangle$ as a quantum superposition of $\left|\Psi_{1}\right\rangle$. In doing so, we introduce an, a priori, arbitrary function $g\left(\sigma ; k_{\mathrm{b}}\right)$ of $\sigma$. The definition of the state $\left|\Psi_{2}\left(n, k_{\mathrm{b}}\right)\right\rangle$

$$
\left|\Psi_{2}\left(n, k_{\mathrm{b}}\right)\right\rangle \equiv \int_{0}^{+\infty} \mathrm{d} \sigma g\left(\sigma ; k_{\mathrm{b}}\right)\left|\Psi_{1}(\sigma, n)\right\rangle,
$$

where $g\left(\sigma ; k_{\mathrm{b}}\right)$ is a given function which defines the privileged scale $k_{\mathrm{b}}$. We assume that the state is normalized and therefore $\int_{0}^{+\infty} g^{2}\left(\sigma ; k_{\mathrm{b}}\right) \mathrm{d} \sigma=1$. In the state $\left|\Psi_{1}(\sigma, n)\right\rangle$, for any domain $\mathcal{D}$ one has

$$
\begin{aligned}
& \left\langle\Psi_{1}(\sigma, n)\left|c_{\mathbf{p}} c_{\mathbf{q}}\right| \Psi_{1}(\sigma, n)\right\rangle=\left\langle\Psi_{1}(\sigma, n)\left|c_{\mathbf{p}}^{\dagger} c_{\mathbf{q}}^{\dagger}\right| \Psi_{1}(\sigma, n)\right\rangle=0, \\
& \left\langle\Psi_{1}(\sigma, n)\left|c_{\mathbf{p}} c_{\mathbf{q}}^{\dagger}\right| \Psi_{1}(\sigma, n)\right\rangle=n \delta(\mathbf{q} \in \mathcal{D}) \delta(\mathbf{p}-\mathbf{q})+\delta(\mathbf{p}-\mathbf{q}), \\
& \left\langle\Psi_{1}(\sigma, n)\left|c_{\mathbf{p}}^{\dagger} c_{\mathbf{q}}\right| \Psi_{1}(\sigma, n)\right\rangle=n \delta(\mathbf{q} \in \mathcal{D}) \delta(\mathbf{p}-\mathbf{q}) .
\end{aligned}
$$

In these formulas, $\delta(\mathbf{q} \in \mathcal{D})$ is a function that is equal to 1 if $\mathbf{q} \in \mathcal{D}$ and 0 otherwise. These relations will be employed in the sequel for the computation of the CMB temperature anisotropies for the different non-vacuum initial states.

\subsection{Two-point function of the CMB temperature anisotropy}

The spherical harmonic expansion of the cosmic microwave background temperature anisotropy, as a function of angular position, is given by

$$
\frac{\delta T}{T}(\mathbf{e})=\sum_{\ell m} a_{\ell m} Y_{\ell m}(\mathbf{e}) \quad \text { with } \quad a_{\ell m}=\int \mathrm{d} \Omega_{\mathbf{e}} \frac{\delta T}{T}(\mathbf{e}) Y_{\ell m}^{*}(\mathbf{e}) .
$$

As we are interested in a non-Gaussian signature of primordial origin we will be focusing on large angular scales, for which the main contribution to the temperature anisotropy is given by the Sachs-Wolfe effect, namely, $\delta T / T(\mathbf{e}) \simeq(1 / 3) \Phi\left[\eta_{\mathrm{lss}}, \mathbf{e}\left(\eta_{0}-\right.\right.$ $\left.\eta_{\text {lss }}\right)$ ], where $\Phi(\eta, \mathbf{x})$ is the Bardeen potential, while $\eta_{0}$ and $\eta_{\text {lss }}$ denote respectively the conformal times now and at the last scattering surface. Note that the previous expression is only valid for the standard Cold Dark Matter model (sCDM). In general, we might also be interested in the case where a cosmological constant is present $(\Lambda \mathrm{CDM}$ model) since this seems to be favored by recent observations. Then, the integrated Sachs-Wolfe effect plays a non-negligible role on large scales and the expression giving the temperature fluctuations is not as simple as the previous one.

In the theory of cosmological perturbations of quantum mechanical origin, the Bardeen variable becomes an operator, and its expression can be written as

$$
\Phi(\eta, \mathbf{x})=\frac{\ell_{\mathrm{Pl}}}{\ell_{0}} \frac{3}{4 \pi} \int \mathrm{d} \mathbf{k}\left[c_{\mathbf{k}}\left(\eta_{\mathrm{i}}\right) f_{k}(\eta) e^{i \mathbf{k} \cdot \mathbf{x}}+c_{\mathbf{k}}^{\dagger}\left(\eta_{\mathrm{i}}\right) f_{k}^{*}(\eta) e^{-i \mathbf{k} \cdot \mathbf{x}}\right],
$$


where $\ell_{\mathrm{Pl}}=(G \hbar)^{1 / 2}$ is the Planck length. In the following, we will consider the class of models of power-law inflation since the power spectrum of the fluctuations is then explicitly known. In this case, the scale factor reads $a(\eta)=\ell_{0}|\eta|^{1+\beta}$, where $\beta \leq-2$ is a priori a free parameter. However, in order to obtain an almost scaleinvariant spectrum, $\beta$ should be close to -2 . In the previous expression of the scale factor, the quantity $\ell_{0}$ has the dimension of a length and is equal to the Hubble radius during inflation if $\beta=-2$. The parameter $\ell_{0}$ also appears in Eq. (8). The mode function $f_{k}(\eta)$ of the Bardeen operator is related to the mode function $\mu_{k}(\eta)$ of the perturbed inflaton through the perturbed Einstein equations. In the case of power-law inflation and in the long wavelength limit, the function $f_{k}(\eta)$ is given in terms of the amplitude $A_{\mathrm{S}}$ and the spectral index $n_{\mathrm{s}}$ of the induced density perturbations by

$$
k^{3}\left|f_{k}\right|^{2}=A_{\mathrm{s}} k^{n_{\mathrm{s}}-1} .
$$

Using the Rayleigh equation and the completeness relation for the spherical harmonics and after some algebra we get

$$
a_{\ell m}=\frac{\ell_{\mathrm{Pl}}}{\ell_{0}} e^{i \pi \ell / 2} \int \mathrm{d} \mathbf{k}\left[c_{\mathbf{k}}\left(\eta_{\mathrm{i}}\right) f_{k}(\eta)+c_{-\mathbf{k}}^{\dagger}\left(\eta_{\mathrm{i}}\right) f_{k}^{*}(\eta)\right] j_{\ell}\left[k\left(\eta_{0}-\eta_{\mathrm{lss}}\right)\right] Y_{\ell m}^{*}(\mathbf{k}) .
$$

At this point we need to somehow restrict the shape of the domain $\mathcal{D}$. We assume that the domain only restricts the modulus of the vectors, while it does not act on their direction. Then, from Eq. (10), one deduces

$$
\begin{gathered}
\left\langle\Psi_{1}(\sigma, n)\left|a_{\ell_{1} m_{1}} a_{\ell_{2} m_{2}}^{*}\right| \Psi_{1}(\sigma, n)\right\rangle=\frac{\ell_{\mathrm{Pl}}^{2}}{\ell_{0}^{2}}\left[C_{\ell_{1}}+2 n D_{\ell_{1}}^{(1)}(\sigma)\right] \delta_{\ell_{1} \ell_{2}} \delta_{m_{1} m_{2}}, \\
D_{\ell}^{(1)}(\sigma) \equiv \int_{0}^{\sigma} j_{\ell}^{2}\left[k\left(\eta_{0}-\eta_{\mathrm{lss}}\right)\right] k^{3}\left|f_{k}\right|^{2} \frac{\mathrm{d} k}{k}
\end{gathered}
$$

Thus, the multipole moments $C_{\ell}^{(1)}$, in the state $\left|\Psi_{1}\right\rangle$, are given by $C_{\ell}^{(1)}(\sigma)=C_{\ell}+$ $2 n D_{\ell}^{(1)}(\sigma)$, where $C_{\ell}$ is the "standard" angular power spectrum, i.e., the multipole obtained in the case where the quantum state is the vacuum, i.e., $n=0$. Let us calculate the same quantity in the state $\left|\Psi_{2}\right\rangle$. Performing a similar analysis as the above one, we find $[7$

$$
\begin{gathered}
\left\langle\Psi_{2}\left(n, k_{\mathrm{b}}\right)\left|a_{\ell_{1} m_{1}} a_{\ell_{2} m_{2}}^{*}\right| \Psi_{2}\left(n, k_{\mathrm{b}}\right)\right\rangle=\frac{\ell_{\mathrm{Pl}}^{2}}{\ell_{0}^{2}}\left[C_{\ell_{1}}+2 n D_{\ell_{1}}^{(2)}\right] \delta_{\ell_{1} \ell_{2}} \delta_{m_{1} m_{2}}, \\
D_{\ell}^{(2)}=\frac{\pi}{2} A_{\mathrm{S}} \int_{0}^{+\infty} J_{\ell+1 / 2}^{2}(k) \bar{h}(k) k^{n_{\mathrm{S}}-3} \mathrm{~d} k
\end{gathered}
$$

where, to reach this eqn, we defined $g^{2}\left(\sigma ; k_{\mathrm{b}}\right) \equiv \mathrm{d} h / \mathrm{d} \sigma$ [we will see below that this function $h\left(k_{\mathrm{b}}\right)$ cannot be arbitrary] and we integrated by parts, and then we defined $\bar{h}(k) \equiv h(\infty)[1-h(k) / h(\infty)]$. In this we have not assumed anything on $h(\infty)$ or $h(0)$. We see that the relation $g^{2}(k) \equiv \mathrm{d} h / \mathrm{d} k$ requires the function $h(k)$ to be monotonically increasing with $k$. It is interesting that, already at this stage of the calculations, very stringent conditions are required on the function $h(k)$ which is therefore not arbitrary. This implies that the function $\bar{h}(k)$ which appears in the 
correction to the multipole moments is always positive, vanishes at infinity and is monotonically decreasing with $k$. An explicit profile for $\bar{h}(k)$ is given in Fig. 10 total power spectrum of the Bardeen potential can be written as

$$
k^{3}\left|\Phi_{k}\right|^{2} \propto A_{\mathrm{S}} k^{n_{\mathrm{S}}-1}\left\{1+2 n h(\infty)\left[1-\frac{h(k)}{h(\infty)}\right]\right\}=A_{\mathrm{S}} k^{n_{\mathrm{S}}-1}[1+2 n \bar{h}(k)] .
$$

Observations indicate that $n_{\mathrm{S}} \simeq 1$ and for simplicity we will take $n_{\mathrm{S}}=1$. As we have seen previously, we can write the multipole moments in the state $\left|\Psi_{2}\right\rangle$ as $C_{\ell}^{(2)}=C_{\ell}+2 n D_{\ell}^{(2)}$. Substituting the well-known expression for the $C_{\ell}$ 's and the definition of $D_{\ell}^{(2)}$ given by Eq. (14), one finds that the coefficients $C_{\ell}^{(2)}$ are given by

$$
C_{\ell}^{(2)}=A_{\mathrm{s}} \frac{\pi}{2}\left\{\frac{1}{2^{3-n_{\mathrm{s}}}} \frac{\Gamma\left(3-n_{\mathrm{s}}\right) \Gamma\left[\ell+\left(n_{\mathrm{s}}-1\right) / 2\right]}{\Gamma^{2}\left[\left(4-n_{\mathrm{s}}\right) / 2\right] \Gamma\left[\ell-\left(n_{\mathrm{s}}-5\right) / 2\right]}+2 n \bar{D}_{\ell}^{(2)}\right\} .
$$

As a next step, one has to normalize the spectrum (need to determine the value of $\left.A_{\mathrm{S}}\right)$. We choose to use the value of $Q_{\mathrm{rms}-\mathrm{PS}}=T_{0}\left[5 C_{2}^{(2)} /(4 \pi)\right]^{1 / 2}\left(\ell_{\mathrm{Pl}} / \ell_{0}\right) \sim 18 \mu K$ with $T_{0}=2.7 \mathrm{~K}$ measured by the COBE satellite. Thus, we compute the quadrupole and then

$$
A_{\mathrm{S}}=\frac{8}{5} \frac{Q_{\mathrm{rms}-\mathrm{PS}}^{2}}{T_{0}^{2}} \frac{\ell_{0}^{2}}{\ell_{\mathrm{Pl}}^{2}}\left[\frac{1}{6 \pi}+2 n \bar{D}_{2}^{(2)}\right]^{-1},
$$

for $n_{\mathrm{S}}=1$. The band power $\delta T_{\ell}$ gives

$$
\delta T_{\ell}=\frac{Q_{\mathrm{rms}-\mathrm{PS}}}{T_{0}} \sqrt{\frac{12}{5}} \sqrt{\frac{1+2 n \pi \ell(\ell+1) \bar{D}_{\ell}^{(2)}}{1+12 n \pi \bar{D}_{2}^{(2)}}} .
$$

The $n$-dependence in the above expression is the correction due to the non-vacuum initial state. We easily check that if $n=0$ the corresponding band powers are constant at large angular scales.

Finally, we calculate the two-point correlation function at zero lag in the state $\left|\Psi_{2}\right\rangle$. Using Eqs. (77), (13), the second moment, $\mu_{2}$, of the distribution is given by

$$
\mu_{2} \equiv\left\langle\left[\frac{\delta T}{T}(\mathbf{e})\right]^{2}\right\rangle=\frac{\ell_{\mathrm{Pl}}^{2}}{\ell_{0}^{2}} \sum_{\ell} \frac{2 \ell+1}{4 \pi} C_{\ell}^{(2)} .
$$

Once we have reached this point, an obvious first thing to do is to check that the twopoint correlation function calculated above is consistent with present observations.

\subsection{Comparison with observations}

Among the available observations that one can use to check the predictions of theoretical models, two are key in cosmology: the CMB anisotropy and the matterdensity power spectra. We will not study in details all the predictions that can be done from the two-point correlation function since our main purpose in this note is to calculate the non-Gaussianity which is a clear specific signature of a non 

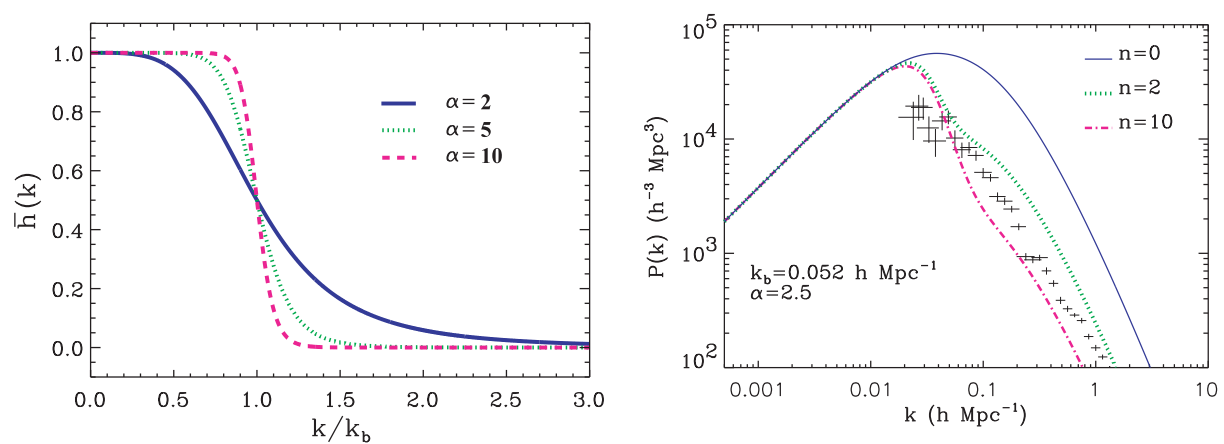

Fig. 1. The function $\bar{h}(k)$ for different values of $\alpha$ (left panel). On the right panel we show the matter power spectrum normalized to COBE for different numbers $n$ of quanta in the initial state. The cosmological parameters are those corresponding to the sCDM model, namely, $h=0.65$, $\Omega_{\Lambda}=0, \Omega_{\mathrm{b}}=0.05, \Omega_{\mathrm{cdm}}=0.95$ and $n_{\mathrm{S}}=1$. The parameters describing the non vacuum state are $k_{\mathrm{b}}^{\text {phys }}=0.052 h \mathrm{Mpc}^{-1}$ and $\alpha=2.5$. The data points represent the power spectrum measured by the PSCz survey.

vacuum state. So, we just compute the matter power spectrum to demonstrate that it fits reasonably well the available astrophysical observations for some values of the free parameters. In addition, this illustrates well the fact that, using the available observations, we can already put some constraints on the free parameters. A simple ansatz for the function $\bar{h}(k)$ is represented in Fig. 䧃and can be expressed as

$$
\bar{h}(k)=\frac{1}{2}\left[1-\tanh \left(\alpha \ln \frac{k}{k_{\mathrm{b}}}\right)\right] .
$$

With it, the matter power spectrum today, after taking into account the transfer function $T(k)$ which describes the evolution of the Fourier modes inside the horizon, can be written

$$
P(k)=T^{2}(k) \frac{16 \pi}{5 H_{0}^{4}} \frac{Q_{\mathrm{rms}-\mathrm{PS}}^{2}}{T_{0}^{2}}\left[\frac{1}{6 \pi}+2 n \bar{D}_{2}^{(2)}\left(k_{\mathrm{b}}\right)\right]^{-1}[1+2 n \bar{h}(k)] k_{\mathrm{phys}} .
$$

The sCDM transfer function is given approximatively by the numerical fit 5

$$
T(k)=\frac{\ln (1+2.34 q)}{2.34 q}\left[1+3.89 q+(16.1 q)^{2}+(5.46 q)^{3}+(6.71 q)^{4}\right]^{-1 / 4},
$$

where $q$ and the shape parameter $\Gamma$ can be written as

$$
q \equiv k /\left[(h \Gamma) \mathrm{Mpc}^{-1}\right] \quad \Gamma \equiv \Omega_{0} h e^{-\Omega_{\mathrm{b}}-\sqrt{2 h} \Omega_{\mathrm{b}} / \Omega_{0}},
$$

where $\Omega_{0}$ is the total energy density to critical energy density ratio and $\Omega_{\mathrm{b}}$ represents the baryon contribution. Or, more explicitly, we take $\Omega_{0}=\Omega_{\Lambda}+\Omega_{\mathrm{m}}=$ $\Omega_{\Lambda}+\Omega_{\mathrm{cdm}}+\Omega_{\mathrm{b}}$. We have now normalized the matter power spectrum to COBE. It is important to realize that the above procedure only works for the SCDM model since we have used the Sachs-Wolfe equation. The sCDM matter power spectrum is depicted in Fig. 1 The measured power spectrum of the IRAS Point Source 
Catalogue Redshift Survey (PSCz) $\underline{6}$ has also been displayed for comparison. One notices that the effect of the step in $\bar{h}(k)$ is to reduce the power at small scales which precisely improves the agreement between the theoretical curves for $n \neq 0$ and the data. Let us remind at this point that the shape of the function $\bar{h}(k)$ has not been designed for this purpose and comes from different (theoretical) reasons. Therefore, it is quite interesting to see that the power spectrum obtained from our ansatz fits reasonably well the data. This plot also confirms the result of Ref. 2, namely that the number of quanta must be such that (for sCDM) $1 \leq n<10$, i.e., it cannot be too large.

\section{Four-point correlation function for non-vacuum initial states}

It is time to proceed with the calculation of the four-point correlation function. Briefly, one has to first perform the calculation for the state $\left|\Psi_{1}\right\rangle$, and then generalize it for $\left|\Psi_{2}\right\rangle$. After some lengthy but straightforward algebra, one finds ${ }^{7}$

$$
\begin{gathered}
\left\langle\Psi_{2}\left(n, k_{\mathrm{b}}\right)\left|a_{\ell_{1} m_{1}} a_{\ell_{2} m_{2}} a_{\ell_{3} m_{3}} a_{\ell_{4} m_{4}}\right| \Psi_{2}\left(n, k_{\mathrm{b}}\right)\right\rangle=\frac{\ell_{\mathrm{P}}^{4}}{\ell_{0}^{4}}\{ \\
(-1)^{m_{1}+m_{2}}\left[C_{\ell_{1}} C_{\ell_{2}}+2 n C_{\ell_{1}} D_{\ell_{2}}^{(2)}+2 n C_{\ell_{2}} D_{\ell_{1}}^{(2)}+4 n^{2} F_{\ell_{1} \ell_{2}}^{(2)}\right] \delta_{\ell_{1} \ell_{3}} \delta_{\ell_{2} \ell_{4}} \delta_{m_{1},-m_{3}} \delta_{m_{2},-m_{4}} \\
+(-1)^{m_{1}+m_{2}}\left[C_{\ell_{1}} C_{\ell_{2}}+2 n C_{\ell_{1}} D_{\ell_{2}}^{(2)}+2 n C_{\ell_{2}} D_{\ell_{1}}^{(2)}+4 n^{2} F_{\ell_{1} \ell_{2}}^{(2)}\right] \delta_{\ell_{1} \ell_{4}} \delta_{\ell_{2} \ell_{3}} \delta_{m_{1},-m_{4}} \delta_{m_{2},-m_{3}} \\
+(-1)^{m_{1}+m_{3}}\left[C_{\ell_{1}} C_{\ell_{3}}+2 n C_{\ell_{1}} D_{\ell_{3}}^{(2)}+2 n C_{\ell_{3}} D_{\ell_{1}}^{(2)}+4 n^{2} F_{\ell_{1} \ell_{3}}^{(2)}\right] \delta_{\ell_{1} \ell_{2}} \delta_{\ell_{3} \ell_{4}} \delta_{m_{1},-m_{2}} \delta_{m_{3},-m_{4}} \\
\left.-2 n(n+1) E_{\ell_{1} \ell_{2} \ell_{3} \ell_{4}}^{(2)} \mathcal{H}_{\ell_{1} \ell_{2} \ell_{3} \ell_{4}}^{m_{1} m_{2} m_{3} m_{4}} e^{i \pi\left(\ell_{1}+\ell_{2}+\ell_{3}+\ell_{4}\right) / 2}\left[1+(-1)^{\ell_{1}+\ell_{3}}+(-1)^{\ell_{2}+\ell_{3}}\right]\right\},(24) \\
F_{\ell_{1} \ell_{2}}^{(2)} \equiv \int_{0}^{+\infty} \mathrm{d} \sigma \bar{h}(\sigma) \frac{\mathrm{d}}{\mathrm{d} \sigma}\left[D_{\ell_{1}}^{(1)} D_{\ell_{2}}^{(1)}\right] \\
E_{\ell_{1} \ell_{2} \ell_{3} \ell_{4}}^{(2)} \equiv \int_{0}^{+\infty} j_{\ell_{1}}\left[k\left(\eta_{0}-\eta_{\mathrm{lss}}\right)\right] \ldots j_{\ell_{4}}\left[k\left(\eta_{0}-\eta_{\mathrm{lss}}\right)\right] \bar{h}(k) k^{3}\left|f_{k}\right|^{4} \frac{\mathrm{d} k}{k}
\end{gathered}
$$

We are now in a position to calculate the CMB excess kurtosis. In order to establish an analytical formula for it, one just needs to use the equation linking $a_{\ell m}$ and $\delta T / T$ and play with the properties of the spherical harmonics. Explicitly, the excess kurtosis is defined as $\mathcal{K} \equiv \mu_{4}-3 \mu_{2}^{2}$, where the second moment has already been introduced and where the fourth moment, $\mu_{4}$, of the distribution is defined as $\mu_{4}=\langle K\rangle$ with $K \equiv\left[\frac{\delta T}{T}(\mathbf{e})\right]^{4}$. An important shortcoming of the previous definition is that the value of $\mathcal{K}$ depends on the normalization. It is much more convenient to work with a normalized (dimensionless) quantity. Therefore, we also define the normalized excess kurtosis as

$$
\mathcal{Q} \equiv \frac{\mathcal{K}}{\mu_{2}^{2}}=\frac{\mu_{4}}{\mu_{2}^{2}}-3
$$

which is the one more commonly used in the literature. We have performed the full 


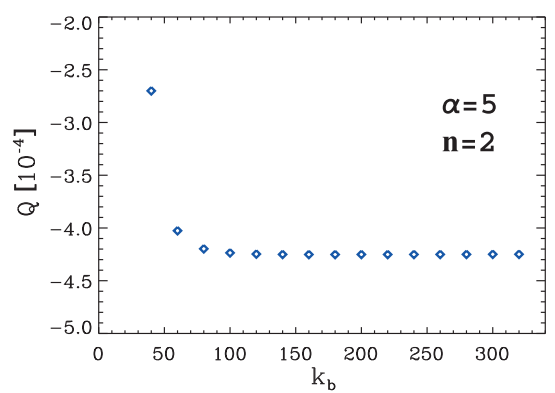

Fig. 2. The normalized excess kurtosis parameter $\mathcal{Q}$ in terms of the privileged (comoving) wavenumber $k_{\mathrm{b}}$ for a particular representative set of parameters: $\alpha=5$ for the sharpness of the weight function signaling the privileged scale, and $n=2$ quanta in the non-vacuum initial state for the cosmological perturbations.

numerical resolution of the normalized excess kurtosis parameter which we show in Fig. [2] As we see from it, $\mathcal{Q} \simeq-4.24 \times 10^{-4}$ is an asymptotic value, provided we concentrate on the middle and big values of the built-in scale $k_{\mathrm{b}}$. An analytical order of magnitude estimate of this can be seen in Ref. 7 . The fact that the numerical estimate does not depend on $k_{\mathrm{b}}$ is confirmed by the plot, except for small values of the wave-numbers. In fact, this shows that the quantity $\mathcal{Q}$ does not depend very much on the free parameters. One can see this is true for parameters $\alpha$ and $n$ since, using the above mentioned analytical estimate, we find, for $n=1, \mathcal{Q} \simeq-3.77 \times 10^{-4}$ and for $n \rightarrow \infty, \mathcal{Q} \simeq-4.25 \times 10^{-4}$. Since we know that this result does not depend on the details of the weight function $\bar{h}(k)$, we conclude that the asymptotic value obtained above is a generic value, at least for large values of $k_{\mathrm{b}}$. In particular, this is true for $k_{\mathrm{b}} \approx 300$ which corresponds to the built-in scale located roughly at the privileged scale in the matter power spectrum selected by the redshift surveys of Ref. 4. Another important remark is that the excess kurtosis is found to be negative.

\section{Discussion}

The above paragraphs show that the introduction of non-vacuum initial states leads to a clear prediction for the excess kurtosis parameter. However, this by itself does not imply that the signature will be observable. The signal to theoretical noise ratio has to be considered or, stating it differently, we need to make the comparison of the signal against the cosmic variance. The cosmic variance quantifies the theoretical error coming from the fact that, in cosmology, observers have only access to one realization of the $\delta T / T$ stochastic process whereas theoretical predictions are expressed through ensemble averages. The specific computation of the cosmic variance for the model under consideration was performed ${ }^{[7]}$ and found to be some four orders of magnitude higher than the signal itself. This is no surprise: we are interested in a non-Gaussian signature of primordial origin, and thus we are focusing on large angular scales, for which the theoretical uncertainties are the highest. Shifting to intermediate angular scales, a stronger signal would be obtained; how- 
$8 \quad$ Alejandro Gangui

ever, in that case secondary sources would be more difficult to subtract and thus the transparency of the effect would be compromised.

In sum, the excess kurtosis is found to be negative and the signal to noise ratio for the dimensionless excess kurtosis parameter is equal to $|S / N| \simeq 4 \times 10^{-4}$, almost independently of the free parameters of the model. This signature turns out to be undetectable and therefore we conclude that, subject to current tests, Gaussianity is a generic property of single field inflationary models.

\section{Acknowledgements}

I'd like to thank my collaborators Jérôme Martin and Mairi Sakellariadou for extensive discussions and for hospitality in Paris. This work was partially financed with funds from CONICET, UBA, IAP and FundaCión ANTORCHAS.

\section{References}

1. L. P. Grishchuk and J. Martin, Phys. Rev. D56, 1924 (1997), gr-qc/9702018

2. J. Martin, A. Riazuelo and M. Sakellariadou, Phys. Rev. D 61, 083518 (2000).

3. L. M. Griffiths, J. Silk and S. Zaroubi, Mon. Not. R. Astron. Soc. 324, 712 (2001).

4. J. Einasto et al., Nature 385, 139 (1997); J. A. Peacock, Mon. Not. R. Astron. Soc. 284, 885 (1997); T. J. Broadhurst et al., Nature 343, 726 (1990).

5. N. Sugiyama, Astrophys. J. Suppl. 100, 281 (1995).

6. A. J. S. Hamilton and M. Tegmark, astro-ph/0008392

7. A. Gangui, J. Martin and M. Sakellariadou, Phys. Rev. D 66, 083502 (1-23) (2002). astro-ph/0205202 